\title{
Optical Negative-Index Metamaterial Using CSRR by Incidenting the Light Horizontally at Near-Infrared Domain
}

\author{
Ammar Armghan, Xinguang Hu, Shuai Yuan, Jinsong Xia* \\ Wuhan National Laboratory for Optoelectronics and School of Optical and Electronic Information, Huazhong \\ University of Science and Technology, Wuhan, China \\ Email: ammar.armghan@gmail.com, ${ }^{*}$ jsxia@hust.edu.cn
}

Received 20 December 2015; accepted 27 March 2016; published 30 March 2016

Copyright (C) 2016 by authors and Scientific Research Publishing Inc.

This work is licensed under the Creative Commons Attribution International License (CC BY). http://creativecommons.org/licenses/by/4.0/

cC) (7) Open Access

\begin{abstract}
Metamaterial structure based on cascaded split ring resonators (CSRR) is proposed in order to produce a negative refractive index in terahertz regime at near-infrared range. We have incident light horizontally instead of incidenting it perpendicular. We have measured the negative refractive index, permeability and permittivity by using the S-parameter analysis. Furthermore, it is found out that negative refractive index, permeability and permittivity are dependent upon the width of the wire and the gap between resonators at near-infrared range. This work will be helpful for the fabrication and design of double negative metamaterials structure having negative permeability, permittivity and negative refractive index for in plane applications.
\end{abstract}

Keywords

Metamaterials, Negative Refractive Index, Left Handed Material, Scattering Parameters

\section{Introduction}

Negative refractive index (NRI) is the main focus point of current research. Within five years after Smith's proposal, the field of NRI has seen development at optical range by using different structures like paired nano-rods [1], nano-fishnet with circular voids [2], nano-fishnet with elliptical voids [3], and nano-fishnet with rectangular voids [4].

As compared to photonic crystals, which retain a periodicity in the order of the wavelength, the size and the distance of the distinct presence in metamaterials are subwavelength. This permits to define the optical response

${ }^{*}$ Corresponding author.

How to cite this paper: Armghan, A., Hu, X.G., Yuan, S. and Xia, J.S. (2016) Optical Negative-Index Metamaterial Using CSRR by Incidenting the Light Horizontally at Near-Infrared Domain. Journal of Electromagnetic Analysis and Applications, 8, 5661. http://dx.doi.org/10.4236/jemaa.2016.83006 
of metamaterials by effective material parameters. This is an equivalence to wave propagation in conventional materials, where the interaction of an electromagnetic wave with a group of atoms or molecules is defined by permittivity $\varepsilon$ and the permeability $\mu$. Effective permittivity $\varepsilon$ eff and an effective permeability $\mu$ eff define the electromagnetic response of the metamaterial. The effective refractive index neff and the effective wave impedance zeff can be calculated from the effective permittivity $\varepsilon$ eff and an effective permeability $\mu$ eff is used to describe the propagation of a wave through the metamaterial.

An array of thin conducting wires, showing an effective plasmonic response at $\mathrm{GHz}$ frequencies [5] and the material composed of split-ring resonators (SRRs) which had a magnetic response [6], was the two basics metamaterial designs that were explained in late 1990s. In 2006, the electric LC-resonator (ELC) with an electric resonance was presented as a third basic metamaterial element [7]. Nowadays, the metamaterial structures evolved on the variations in these three designs. Subsequently for the implementation of optical components, metamaterials have appeared as a flexible device. As compared with conventional materials, the advantage of metamaterials is that the optical response of a metamaterial can be effortlessly designed to the desired values for an application by altering the size and shape of the subwavelength elements. Metamaterials in $\mathrm{THz}$ regime are being used as absorbers [8], quarter waveplates [9], switches and modulators [10]-[15]. Metamaterial negative refractive index has numerous applications such as M-NRI for antennas [16], Superlens [17], and wireless power transfer [18] and for biomedical applications [19] [20].

Since Smith et al. [21] experimentally confirmed the presence of corresponding negative index materials. In the last decade, negative indexed materials (NIMs) or double negative materials (DNG) or left-handed materials (LHMs) with simultaneously negative permittivity $\varepsilon$ and negative permeability $\mu$ have attracted considerable attention. The terahertz $(\mathrm{THz})$ and optical metamaterials fascinated some devotion in recent years. In numerous design techniques of $\mathrm{THz}$ and optical metamaterials, though, the resonant cells should be made much smaller than the operation wavelength. Consequently, problems in fabrication of such small resonant elements and material loss turn out to be big challenges in reality. Furthermore, most of the metamaterials designs are restricted by narrow bandwidths, so tunability of operating frequencies becomes an important issue in the metamaterials research, which motivates the present work.

Most of the current metamaterials research is that light incident on the normal direction. But, many researchers are trying to integrate devices with different functions on a single chip. Therefore, metamaterials to work with light in plane propagation direction is necessary for on-chip integration at near-infrared regime. However, few research of in plane light propagation is reported in case of metamaterials. In this paper, we have studied a metamaterial structure for an in plane light propagation and studied its optical properties at near-infrared range.

\section{Design and Simulation}

Different kinds of techniques have been developed in the past few years for extraction of S-parameters. In our simulation we are using the S-parameters extraction technique discussed by Smith et al. [22]. The structure was simulated by FDTD method. Choosing the frequency unit in THz, S-parameter analysis tool was used to extract the S-parameters. Each calculation is based on 100 frequency samples. The unit cell is shown in Figure 1. It consists of three materials: $\mathrm{SiO}_{2}$ as a substrate, $\mathrm{Si}$ as waveguide and split ring resonator made of gold. This structure was simulated for $216 \mathrm{THz}$ frequency range. The thickness of the substrate is $340 \mathrm{~nm}$, the thickness of the waveguide is $220 \mathrm{~nm}$, width of metal wires is $40 \mathrm{~nm}$, length of the metal wires of the outer ring is $230 \mathrm{~nm}$ and the gap between the inner ring and the outer ring is $40 \mathrm{~nm}$.

\section{Results and Discussion}

The calculated wavelength spectra of the structure are shown in Figure 2(a), where the width of the wires is kept at $40 \mathrm{~nm}$. The appearance of the LH behavior can be confirmed by using the extracted effective parameters of the structure, as presented in Figures 2(b)-(d). Obviously, both the effective permeability and permittivity are negative. Consequently, the refractive index has a negative value $(\mathrm{n}<0)$. Moreover, there is a dependence of the structural parameters of the metal wire on the LH behavior. We found that the electromagnetic response of the structure is significantly affected by the width of the continuous wire. Figure 3 shows spectra's of the compound structure by varying the width of the continuous wire. For this purpose, the width of the wire pairs is kept at $40 \mathrm{~nm}$ while the width of the continuous wire is varied from 20 to $40 \mathrm{~nm}$.

As can be seen, resonance is shifted with an increase in width of the continuous wire. To confirm this pheno- 


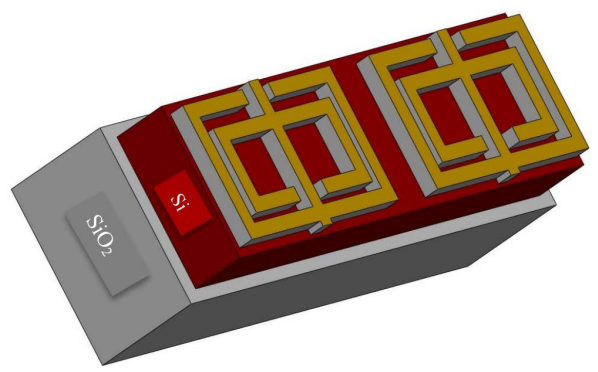

(a)

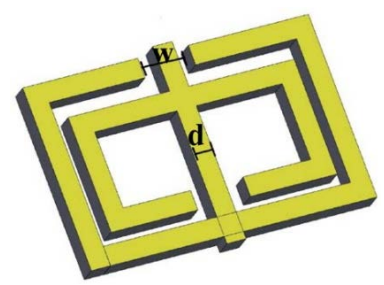

(b)

Figure 1. (a) 3D design of the structure, bottom layer is the $\mathrm{SiO}_{2}$, on top of it is Silicon waveguide and cascaded split ring resonators are placed on the top of the waveguide and (b) shows the parameters width $w$ and the distance $d$ of the cascaded split ring resonators.
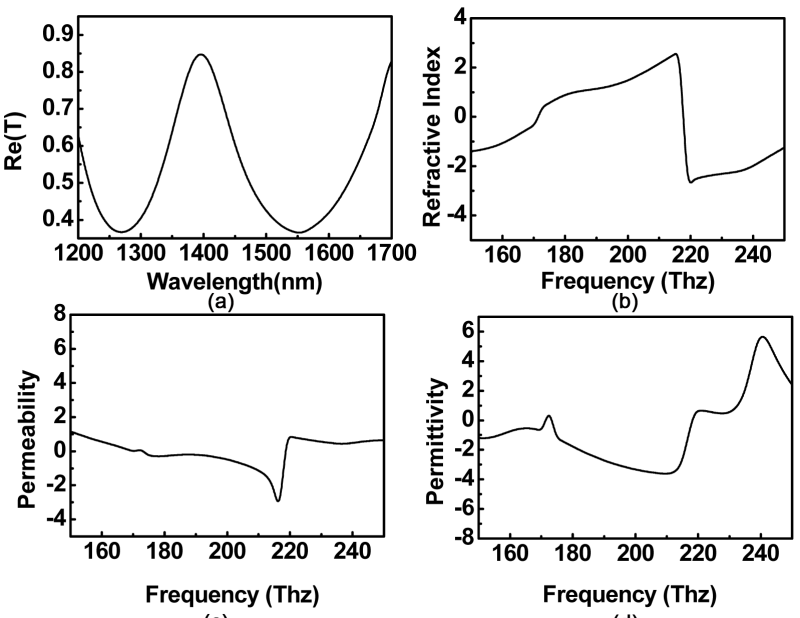

(c)

(d)

Figure 2. (a) Simulated wavelength spectra of the structure, where the width of the wires are kept at $40 \mathrm{~nm}$; ((b)-(d)) real part of the permittivity, permeability and refractive index extracted from the simulation data, respectively.

menon, the effective permittivity, permeability and also refractive index of the structures are extracted from the scattering parameters, as shown in Figures 3(a)-(c), respectively. As expected, the magnetic response shifts towards a lower frequency by decrease in width of the continuous wire, while the plasma frequency remains unchanged. Thus, this explains the reason for the dependence of resonance peak on width of the continuous wire. As Figure 3(a) shows, the refractive index is decreasing with the decrease in width of the continuous wire.

Another interesting result is shown in Figure 4, where the LH behavior of the structure is also intensely affected by varying the gap $d$ between the centers of the continuous wires. To study this phenomenon, the gap $d$ was varied from 30 to $160 \mathrm{~nm}$. The effective permittivity, permeability and refractive index are extracted from simulation results. As it can be seen in the figure, the resonance in the right is shifted by varying the gap $d$ between the centers of the continuous wire. A reduction of negative refractive index appears when decreasing the gap size.

In Table 1, we have compared our results with the other structures near-infrared range that are using perpendicular 


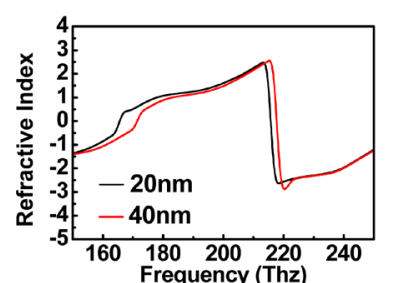

(a)

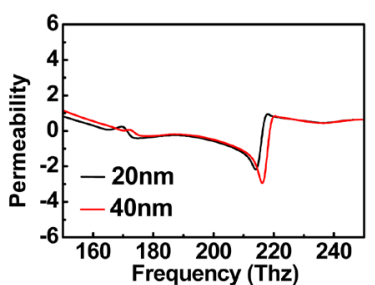

(b)

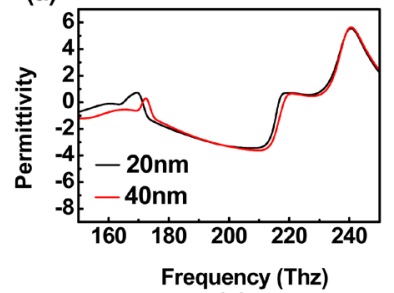

(c)

Figure 3. Simulated spectra of the structure with different widths of the continuous wires. ((a)-(c)) Real part of the refractive index, permeability and permittivity respectively.

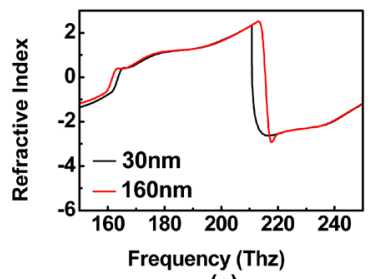

(a)

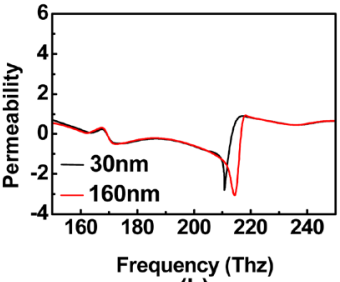

(b)

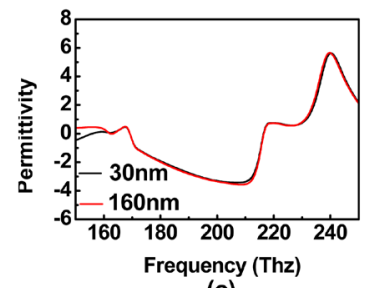

(c)

Figure 4. Extracted effective parameters of structure from the calculated scattering parameters: (a), (b) and (c) are the refractive index, permeability and permittivity, respectively.

Table 1. Comparison between different structures.

\begin{tabular}{|c|c|c|c|c|}
\hline References & Structures & Refractive index & Wavelength & Direction of pump \\
\hline [1] & Paired nanorods & -0.3 & $1.5 \mu \mathrm{m}$ & Perpendicular \\
\hline [23] & Nano-fishnet with circular rods & -2 & $2.0 \mu \mathrm{m}$ & Perpendicular \\
\hline [4] & Nano-fishnet with rectangular rods & -1 & $1.4 \mu \mathrm{m}$ & Perpendicular \\
\hline$[24]$ & Metal-dielectric-metal & -1.05 & $780 \mathrm{~nm}$ & Perpendicular \\
\hline$[25]$ & Multishell nanowires & -2.0 & $690 \mathrm{~nm}$ & Perpendicular \\
\hline Our design [26] & Cascaded split ring resonators & -2.8 & $1.5 \mu \mathrm{m}$ & Horizontally \\
\hline
\end{tabular}


direction of the incident light, where as in our design we are using the horizontal direction of incident light and achieve the refractive index of -2.8 at optical wavelength. The results by using the horizontal direction of incidenting light are useful for designing novel devices for on chip integration by utilizing the optical properties of metamaterials.

\section{Conclusion}

Simulation studies have been conducted on ring-type cascaded split ring resonators (SRR) to understand the properties of metamaterial structure with horizontally incident source at near-infrared range. By incidenting the light along $\mathrm{x}$-axis, the proposed structure shows a negative refractive index indicating the properties of double negative material and left-handed material at near-infrared range. Moreover, it is observed that the width of the continuous wire as well as distance between the centers affects the left-handed behavior of the structure. The search results in our paper are useful for designing novel devices for on chip integration by utilizing the optical properties of metamaterials.

\section{Acknowledgements}

This work was supported by the National “863” Program of China (No. 2015AA016904), the Major State Basic Research Development Program of China (grant 2013CB632104 and 2013CB933303).

\section{References}

[1] Shalaev, V.M., Cai, W.S., Chettiar, U.K., Yuan, H.-K., Sarychev, A.K., Drachev, V.P. and Kildishev, A.V. (2005) Negative Index of Refraction in Optical Metamaterials. Optics Letters, 30, 3356-3358. http://dx.doi.org/10.1364/OL.30.003356

[2] Smith, D.R., Vier, D.C., Koschny, T. and Soukoulis, C.M. (2005) Electromagnetic Parameter Retrieval from Inhomogeneous Metamaterials. Physical Review Letters, 71, 1-11. http://dx.doi.org/10.1103/PhysRevE.71.036617

[3] Zhang, S., Fan W.J., Malloy, K.J., Brueck, S.R., Panoiu, N.C. and Osgood, R.M. (2006) Demonstration of Metal-Dielectric Negative-Index Metamaterials with Improved Performance at Optical Frequencies. Journal of the Optical Society of America, 23, 434-438. http://dx.doi.org/10.1364/JOSAB.23.000434

[4] Dolling, G., Enkrich, C., Wegener, M., Soukoulis, C.M. and Linden, S. (2006) Low-Loss Negative-Index Metamaterial at Telecommunication Wavelengths. Journal of the Optical Society of America, 31, 1800-1802. http://dx.doi.org/10.1364/OL.31.001800

[5] Pendry, J.B., Holden, A.J., Robbins, D.J. and Stewart. W.J. (1998) Low Frequency Plasmons in Thin-Wire Structures. Journal of Physics: Condensed Matter, 10, 4785-4809. http://dx.doi.org/10.1088/0953-8984/10/22/007

[6] Pendry, J.B., Holden, A.J., Robbins, D.J. and Stewart, W.J. (1999) Magnetism from Conductors and Enhanced Nonlinear Phenomena. IEEE Transactions on Microwave Theory and Techniques, 47, 2075-2084. http://dx.doi.org/10.1109/22.798002

[7] Schurig, D., Mock, J.J. and Smith, D.R. (2006) Electric-Field-Coupled Resonators for Negative Permittivity Metamaterials. Applied Physics Letters, 88, 041109. http://dx.doi.org/10.1063/1.2166681

[8] Tao, H., Landy, N.I., Bingham, C.M., Zhang, X., Averitt, R.D. and Padilla, W.J. (2008) A Metamaterial Absorber for the Terahertz Regime: Design, Fabrication and Characterization. Optics Express, 16, 7181-7188. http://dx.doi.org/10.1364/OE.16.007181

[9] Strikwerda, A.C., Fan, K., Tao, H., Pilon, D.V., Zhang, X. and Averitt, R.D. (2009) Comparison of Birefringent Electric Split-Ring Resonator and Meander Line Structures as Quarter-Wave Plates at Terahertz Frequencies. Optics Express, 17, 136-149. http://dx.doi.org/10.1364/OE.17.000136

[10] Chen, H.T., Padilla, W.J., Zide, J.M.O., Gossard, A.C., Taylor, A.J. and Averitt, R.D. (2006) Active Terahertz Metamaterial Devices. Nature, 444, 597-600. http://dx.doi.org/10.1038/nature05343

[11] Chen, H.-T., Padilla, W.J., Cich, M.J., Azad, A.K., Averitt, R.D. and Taylor, A.J. (2009) A Metamaterial Solid-State Terahertz Phase Modulator. Nature Photon, 3, 148-151. http://dx.doi.org/10.1038/nphoton.2009.3

[12] Chen, H.-T., Palit, S., Tyler, T., Bingham, C.M., Zide, J.M.O., O’Hara, J.F., Smith, D.R., Gossard, A.C., Averitt, R.D., Padilla, W.J., Jokerst, N.M. and Taylor, A.J. (2008) Hybrid Metamaterials Enable Fast Electrical Modulation of Freely Propagating Terahertz Waves. Applied Physics Letters, 93, 091117-11091117-3. http://dx.doi.org/10.1063/1.2978071

[13] Chen, H.T., O’Hara, J.F., Azad, A.K., Taylor, A.J., Averitt, R.D., Shrekenhamer, D.B. and Padilla, W.J. (2008) Experimental Demonstration of Frequency-Agile Terahertz Metamaterials. Nature Photon, 2, 295-298. 
http://dx.doi.org/10.1038/nphoton.2008.52

[14] Lapine, M., Powell, D., Gorkunov, M., Shadrivov, I., Marqués, R. and Kivshar, Y. (2009) Structural Tunability in Metamaterials. Applied Physics Letters, 95, 084105-1-084105-3. http://dx.doi.org/10.1063/1.3211920

[15] Tao, H., Strikwerda, A.C., Fan, K., Padilla, W.J., Zhang, X. and Averitt, R.D. (2009) Reconfigurable Terahertz Metamaterials. Physical Review Letters, 103, 147401-1-147401-4. http://dx.doi.org/10.1103/PhysRevLett.103.147401

[16] Chen, X., Ma, H.F., Yang, X., Cheng, Q., Jiang, W.X. and Cui, T.J. (2009) X-Band High Directivity Lens Antenna Realized by Gradient Index Metamaterials. Microwave Conference, Singapore, 7-10 December 2009, 793-797. http://dx.doi.org/10.1109/APMC.2009.5384269

[17] Lier, E. (2011) Metamaterial Lens Feed for Multiple Beam Antennas. United States Patent Application Publication, US 2011/0095953 A1. https://www.google.com/patents/US8576132

[18] Navarro-Cia, M., Beruete, M., Falcone, F., Sorolla, M. and Campillo, I. (2010) Antenna Directivity Enhancement Using a Metamaterial Parabolic Lens. Proceedings of the Fourth European Conference, Barcelona, 12-16 April 2010, 1-3. http://ieeexplore.ieee.org/xpl/login.jsp?tp=\&arnumber=5505200\&url=http\%3A\%2F\%2Fieeexplore.ieee.org\%2Fxpls\% 2Fabs_all.jsp\%3Farnumber

[19] Zhang, X. and Liu, Z.W. (2008) Superlenses to Overcome the Diffraction Limit. Nature Materials, 7, 435-441. http://dx.doi.org/10.1038/nmat2141

[20] Tao, Y.H. and Wang, G. (2012) Conformal Hyperthermia of Superficial Tumor with Left-Handed Metamaterial Lens Applicator. IEEE Transactions on Biomedical Engineering, 59, 3525-3530. http://dx.doi.org/10.1109/TBME.2012.2218108

[21] Smith, D.R., Padilla, W.J., Vier, D.C., Nemat-Nasser, S.C. and Schultz, S. (2000) Composite Medium with Simultaneously Negative Permeability and Permittivity. Physical Review Letters, 84, 4184-4187. http://dx.doi.org/10.1103/PhysRevLett.84.4184

[22] Smith, D.R., Vier, D.C., Koschny, Th. and Soukoulis, C.M. (2005) Electromagnetic Parameter Retrieval from Inhomogeneous Metamaterials. Physical Review Letters, 71, 1-11. http://dx.doi.org/10.1103/PhysRevE.71.036617

[23] Zhang, S, Fan, W.J., Panoiu, N.C., Malloy, K.J., Osgood, R.M. and Brueck, S.R.J. (2005) Experimental Demonstration of Near-Infrared Negative-Index Metamaterials. Physical Review Letters, 95, 137404. http://dx.doi.org/10.1103/PhysRevLett.95.137404

[24] Zhong, M. (2014) Influence of Dielectric Layer on Negative Refractive Index and Transmission of metal-DielectricMetal Sandwiched Metamaterials. Chinese Optics Letters, 12, 041601-1-041601-3. http://dx.doi.org/10.3788/COL201412.041601

[25] Ramadurgam, S. and Yang, C. (2014) Semiconductor-Metal-Semiconductor Core-Multishell Nanowires as NegaticeIndex Metamaterials in Visible Domain. Scientific Reports, 4. http://dx.doi.org/10.1038/srep04931

[26] Armghan, A., Hu, X.G., Yuan, S. and Xia, J.S. (2015) Negative Refractive Index Metamaterial Structure Using SRR by Incidenting the Light Horizontally. Journal of Electromagnetic Analysis and Applications, 7, 276-282. http://dx.doi.org/10.4236/jemaa.2015.711029 